ESTUDIOS

\title{
Luces y sombras: La responsabilidad de la empresa principal en la indemnización especial por tutela laboral con ocasión del despido
}

\author{
Lights and shadows: The responsibility of the main company \\ in the special compensation for labor guardianship on the occasion of dismissal
}

\author{
Tatiana Estefania Saltos Hidalgo \\ Pontificia Universidad Católica del Ecuador
}

\begin{abstract}
RESUMEN La indemnización especial por tutela laboral en el régimen de subcontratación ha implicado una serie de cuestionamientos respecto a la responsabilidad solidaria y subsidiaria de la empresa principal. Para tales efectos se han divisado dos posturas claras, una negacionista y otra que afirma la responsabilidad de la empresa principal. Sin embargo, varios de los criterios que nos propone la tesis negacionista no han sido debidamente cuestionados y, por otro lado, se pueden aportar nuevos enfoques, principalmente desde el derecho constitucional, en favor de la que afirma la responsabilidad por parte de la mandante, acudiendo, entre otros elementos, a los métodos de interpretación y principios propios de esta rama jurídica y sin perder de vista las normas e instituciones pertinentes del Código del Trabajo.
\end{abstract}

PALABRAS CLAVE Subcontratación, tutela laboral, responsabilidad, empresa principal, derechos fundamentales.

ABSTRACT The special compensation for labor guardianship in outsourcing has involved a number of questions regarding the solidary and subsidiary responsibility of the main company. For these purposes two clear positions have been identified, however, several of the criteria proposed by the negationist thesis have not been properly contested and, on the other hand, new approaches can be brought mainly from the Constitutional Law in favor of the one that affirms this responsibility on the part of the principal, coming, among other elements, the methods of interpretation and principles proper to this branch of law and without losing sight of the relevant rules and institutions of the Labour Code.

KEYWORDS Outsourcing, labor guardianship, responsibility, main company, fundamental rights. 


\section{Introducción}

La externalización productiva u outsourcing ha significado un cambio para la estructura empresarial tradicional, a la vez que un desafío para los paradigmas clásicos del derecho laboral desde un enfoque proteccionista o tuitivo del trabajador. No obstante:

Si como lo afirma el profesor Baylos se produce una «huida» del derecho del trabajo con la descentralización productiva en la medida que se desvanece la empresa como centro de imputación de responsabilidades laborales, creemos que ello no debe ser visto como un obstáculo para la legitimidad de la descentralización en tanto se mantenga un respeto a los derechos fundamentales de los trabajadores involucrados en este fenómeno organizativo (Puntriano Rosas, 2011: 153).

En este contexto, un tema que ha suscitado interés es la responsabilidad solidariasubsidiaria de la empresa principal en lo concerniente al pago de la indemnización especial de la tutela laboral con ocasión del despido, contemplado en el artículo 489 inciso tercero del Código del Trabajo. Si algo comparten la doctrina y la jurisprudencia es que no existe claridad sobre las normas que regulan este procedimiento de protección de derechos fundamentales incorporadas en la Ley 20.087, en el contexto de las reglas del régimen de subcontratación incluidas en la Ley 20.123.

Por otro lado, como parte de la metodología de investigación conviene precisar que partiremos esbozando los principales argumentos que cada postura ha expuesto, tanto para presentar una mirada integral de la cuestión a debatir como para realizar las respectivas críticas que dichos razonamientos ameritan, aunque varios de ellos no han tenido lugar en el estadio actual de la materia. Además, se ha estimado necesario conjugar este enfoque dogmático-jurídico con uno jurisprudencial de tipo cualitativo puesto que se busca la construcción de teorías o descubrimientos mediante un método inductivo con base en las premisas recurrentes del órgano jurisdiccional al enfrentarse a esta problemática (Martínez Carazo, 2006: 169). Es relevante afirmar que los fallos representativos que serán objeto de estudio han sido considerados por varios iuslaboralistas, precisamente por la relevancia de su contenido: Lara con Agrícola Concha y Toro, ${ }^{1}$ Rupayan con Team Work Recursos Humanos Ltda. ${ }^{2}$ y Peña con Constructora e Inmobiliaria Casa Grande Limitada. ${ }^{3}$

Existen varias objeciones jurisprudenciales y doctrinarias respecto a que la man-

1. Sentencia del caso Lara con Agrícola Concha y Toro, Corte de Apelaciones de Santiago, rol 455/2011, 5 de julio de 2011 y la sentencia del caso Lara con Concha y Toro, Segundo Juzgado de Letras de Santiago, RIT T-380-2010, 4 de marzo de 2011.

2. Sentencia del caso Rupayan Sandoval, Andrea con Team Work Recursos Humanos Ltda. y otros, Corte de Apelaciones de Concepción, rol 135/2015, 10 de junio de 2015.

3. Sentencia del caso Peña con Constructora e Inmobiliaria Casa Grande Ltda., Juzgado de Letras del Trabajo de Calama, RIT T-13-09, 26 de mayo de 2010. 
dante pueda ser responsable, las mismas que responden a consideraciones de diversa índole, pero varias de ellas radican en una interpretación literal de las normas que rigen la subcontratación, destacando la imposibilidad de aplicarlas en el escenario que nos ocupa. Otros han postulado que existen diferencias en la naturaleza jurídica de las indemnizaciones laborales y previsionales de dar con ocasión del despido y aquella que se contempla para los casos de tutela laboral; así como también han afirmado el carácter personal de los actos u omisiones que se producen en un contexto de vulneración de derechos fundamentales. Además de tales argumentos, hemos recogido otros que pueden ser de utilidad para quienes son adeptos a esta tesis, aunque no son tan comunes o se encuentran ausentes en la motivación de los órganos jurisdiccionales.

Para quienes acogemos una visión diferente, hemos encontrado puntos paralelos - como la interpretación amplia del artículo 183-B del Código del Trabajo-basados en los principios protectorio y de ajenidad. Sin perjuicio de esta clase de razonamientos, se hallan en la periferia otros que no resultan menos interesantes, como la discusión en cuanto a que el pago de la indemnización especial de la tutela laboral constituye propiamente una obligación de dar.

Es así como este artículo propone un análisis crítico de la tesis negacionista y pretende abonar con nuevas perspectivas que apoyan la responsabilidad que se sigue en contra de la empresa principal como consecuencia de la tutela laboral con ocasión del despido, principalmente desde una mirada constitucional de lo laboral, sin perder de vista las normas legales sobre las cuales se desenvuelve este debate. Hacemos esta aclaración sobre todo para quienes ven con cierto escepticismo un eventual argumento relacionado con el principio protectorio.

\section{Tesis negacionista de la responsabilidad de la empresa principal}

Es propicio iniciar este apartado con los puntos más recurrentes que han sido esbozados. Así, si bien se suele rechazar una postura en términos absolutos al respecto, se afirma que la mandante no puede responder por la aplicación del artículo 183-B del Código del Trabajo, toda vez que de su claro tenor se desprende que solo lo será en cuanto a las obligaciones laborales y previsionales de dar, excluyendo de forma expresa e intencional por parte del legislador la indemnización especial prevista en el artículo 489 inciso tercero del mismo cuerpo legal, por constituir una obligación de no hacer, esto es, no vulnerar derechos fundamentales (Palavecino Cáceres, 2014: 35). Este primer criterio refuta la interpretación amplia que se da al artículo 183-B del Código del Trabajo como se produjo, por ejemplo, en el fallo Lara con Agrícola Concha y Toro:

Se trata, como puede apreciarse, de una norma legal de amplio alcance, y los términos generales en que se encuentra redactada permite perfectamente aplicarla a un 
caso como el de la especie, por lo que no existe la infracción legal que se denuncia, cuando se la ha interpretado en un determinado sentido. ${ }^{4}$

Si el lector observó detenidamente, hicimos una salvedad antes de exponer este primer razonamiento y es que, como se deriva de quienes lo desarrollan, no se descarta en principio toda posibilidad de que la empresa principal pueda responder, siempre que hubiera participado directamente en tales actos atentatorios a la dignidad del trabajador. De hecho, hay quienes han precisado que en ese caso sería procedente la aplicación de normas generales de responsabilidad extracontractual por daño moral (Sanhueza Torres, 2015: 193).

Ahora bien, desde la postura contraria se ha rechazado ese argumento indicando que dicha indemnización también es una de dar; debemos coincidir en que, por su origen, por decirlo de alguna manera, sí se refiere a una obligación de no hacer. De forma paralela, si tomamos como punto de partida la tríada de obligaciones que son exigibles al Estado respecto a los derechos humanos - respetar, proteger y garantizar- y que, a la vez, en su rol como primer garante por ser su principal infractor, se ha entendido su obligación de respetarlos en el sentido de una abstención de no violarlos o perturbarlos de manera que haga ineficaz su goce, se puede señalar que esta interpretación es aplicable a los particulares igualmente. Por eso, aceptamos que resulta ciertamente cuestionable que se conciba como una obligación de dar, aunque eso no impide que esta idea sea igual de cuestionable como lo es el siguiente argumento de esta tesis: la diferente naturaleza jurídica que se contempla en las indemnizaciones de los artículos 183-B y 489 del Código del Trabajo.

En segundo término, se sostiene que la indemnización legal por término del contrato no tiene el carácter de sanción:

[Ya que es] un mecanismo contemplado en la legislación laboral para atenuar los perjudiciales efectos que tiene para el trabajador perder su fuente de ingresos, no siendo el comportamiento del empleador la razón por la cual el trabajador tiene derecho de recibir este pago. ${ }^{5}$

De otra parte, el pago que establece el artículo 489 inciso tercero del mismo cuerpo legal operaría como sanción para el sujeto que lesiona ciertos derechos fundamentales del trabajador, al igual que una compensación por el menoscabo sufrido, independiente de que se deba junto con una indemnización legal por término de la relación laboral. Este argumento ha sido compartido por Urzúa (2018: 286-287), al decir:

4. Sentencia del caso Lara con Agrícola Concha y Toro, Corte de Apelaciones de Santiago, rol 455/2011, 5 de julio de 2011.

5. Sentencia del caso Rupayan Sandoval, Andrea con Team Work Recursos Humanos Ltda. y otros, Corte de Apelaciones de Concepción, rol 135/2015, 10 de junio de 2015. 
La naturaleza jurídica de la indemnización-sanción-especial por tutela de derechos fundamentales establecida en el artículo 489 del Código del Trabajo, detenta una naturaleza sancionatoria y no indemnizatoria, respecto de la empresa principal.

Este mismo enfoque lo notamos en la sentencia Rupayan con Team Work Recursos Humanos Ltda. ${ }^{6} \mathrm{Al}$ señalarse, de forma precisa:

El artículo $183-\mathrm{B}$ ya referido es claro en cuanto disponer que tal condena solidaria procede solo en el pago de las indemnizaciones legales con ocasión del término de un contrato de trabajo, sin referirse a las sanciones por vulneración de derechos, omisión realizada precisamente porque las indemnizaciones por término del contrato de trabajo y las sanciones por vulneración de derechos fundamentales tienen una naturaleza jurídica distinta. La indemnización legal por término del contrato de trabajo es una suma de dinero que debe pagarse al trabajador en los casos en que el empleador lo despide por alguna de las causales establecidas en el artículo 161 del Código del Trabajo, si es que el contrato de trabajo ha estado vigente durante un año o más. [...] Por otra parte, el pago que contempla el artículo 489 del Código del Trabajo es una sanción para aquellos sujetos que vulneran ciertas garantías constitucionales del trabajador.

Es evidente que se puede tener varios reparos sobre este punto, pero no se hará la distinción ya que el eje de esa tesis es una interpretación literal de la norma, lo que es una clara contradicción con el aforismo jurídico donde la ley no distingue, no le cabe al intérprete distinguir, por ende, un juicio basado en una eventual diferenciación entre ambas indemnizaciones no cabría atendiendo que el artículo 489 inciso tercero del mismo cuerpo normativo utiliza expresamente el término indemnización.

Tampoco coincidimos debido a que, pese a lo usual de su comprensión contraria, las indemnizaciones previstas en el ordenamiento jurídico por el término del contrato no se traducen en un mero alivio económico para el trabajador ya que, si lo fueran, no variarían conforme a la modalidad de despido o desvinculación que se produzca. Es decir, no serían las mismas si se trata de un despido injustificado o uno por caso fortuito o fuerza mayor, por ejemplo.

Incluso sosteniendo que la indemnización es una sanción, no deja de tener un efecto indemnizatorio debido a que significa una compensación por el perjuicio causado en contra del trabajador, como bien lo reconoce implícitamente este mismo argumento, aunque en el escenario de la vulneración de derechos fundamentales nos refiramos a un daño extrapatrimonial.

Como siguiente objeción se plantea que la afectación a derechos constitucionales es de carácter personalísimo con relación a quien realiza dichos actos, lo que lleva a reafirmar la excepción de responsabilidad que formula esta tesis, como señalamos

6. Sentencia del caso Rupayan Sandoval, Andrea contra Team Work Recursos Humanos Ltda. y otros. 
en un inicio (Sanhueza Torres, 2015: 191-192). Esta postura fue adoptada en Peña con Constructora e Inmobiliaria Casa Grande Limitada ${ }^{7}$ al decir:

Que en primer lugar el vulnerar derechos fundamentales es un actuar personal y directo de responsabilidad únicamente de quien realiza los actos vulneratorios y segundo de conformidad a las normas de subcontratación la responsabilidad de la empresa mandante solo dice relación con obligaciones laborales y previsionales, que se generan como consecuencia de la prestación de servicios en los términos del artículo 7 del Código del Trabajo y no como sanción a ilícitos laborales.

Sin embargo, y pese a no compartir este análisis, este criterio resulta interesante desde la lógica constitucional por varios aspectos. En este sentido, se ha manifestado:

El artículo 485 inciso 3 del Código del Trabajo tipifica un ilícito laboral objetivo, al igual que lo hace el artículo 2 inciso 3 de este cuerpo legal al conceptualizar los actos de discriminación, por lo que para su consumación no se debe atender a aspectos volitivos del comportamiento del empleador, en concreto, a la culpa o dolo implícita en el desarrollo de conducta (Caamaño Rojo, 2007: 189).

La limitación que se pretende en la legitimación pasiva de esta acción no es concordante con la generalidad de los derechos fundamentales, como sería el caso de los derechos económicos, sociales, culturales y ambientales, ya que en su vulneración resulta atinente la posibilidad de que el presunto responsable sea solamente determinable y no concreto al momento que se formula la pretensión, constatada la vulneración a la garantía constitucional.

Adoptar este criterio no solo excluye prima facie la responsabilidad de la mandante, sino a toda empresa respecto de sus trabajadores - sea dentro o fuera del régimen de subcontratación-, ya que la concepción clásica de la relación empleador-trabajador en la que inicialmente se consolidó el derecho del trabajo, y que se presume aquí, hoy dista diametralmente de su origen dado lo dinámico de las relaciones laborales, que ha hecho que por la compleja estructura empresarial interna muchos trabajadores no lleguen a conocer a su empleador, a veces ni siquiera para efectos de suscribir su contrato, pues es probable que solo tengan contacto con un agente o lo hagan a través de medios tecnológicos. ${ }^{8}$ En definitiva, en el escenario actual creemos que no sería exitoso este argumento propuesto por la tesis negacionista.

En este punto es interesante el planteamiento de César Puntriano Rosas (2011:157), al describir la injerencia natural que existe por parte de la empresa principal:

7. Sentencia del caso Lara Peña con Constructora e Inmobiliaria Casa Grande Ltda., Juzgado de Letras del Trabajo de Calama, RIT T-13-09, 26 de mayo de 2010. De forma similar se resuelve en la sentencia del caso Lara con Chiyong y Antonucci con Administradora Casagrande S.A. (Manresa, 2016: 37-38).

8. Algunos de ellos incluso han sido denominados «invisibles», como los trabajadores de las plataformas digitales. «Los invisibles: Plataformas digitales y explotación laboral», Deutsche Welle (2021). 
En efecto, al existir más de una empresa involucrada en este fenómeno productivo (principal y subcontratistas) lo natural es que sea la empresa principal la [que] mantenga el control del ciclo productivo externalizado en las subcontratas toda vez que su presencia en el mercado le exige mantener ciertos estándares que, al haber transferido actividades productivas a sus proveedores de servicios, sea a estos a quienes les requiera su cumplimiento. [...] Inclusive, no puede negarse que ocurren casos en que la empresa principal emite directrices que condicionan el desempeño laboral del personal asignado al servicio, tales como reglas de conducta o pautas de seguridad ocupacional, exigencias de cumplimiento de horarios de trabajo, llegando a condicionar las mejoras salariales del personal del tercero en función al presupuesto asignado al servicio.

Adicionalmente, se ha manifestado que las normas que regulan la tutela laboral son claras al determinar que la lesión de derechos fundamentales debe producirse como consecuencia del ejercicio de facultades de mando, ${ }^{9}$ ergo, dentro de una relación de trabajo, lo que no sucedería en el caso de la empresa principal y los trabajadores de la contratista o subcontratista. De igual manera, Urzúa (2018: 283) indica:

La empresa principal no vulnera directamente los derechos fundamentales de los trabajadores en régimen de subcontratación pues no es empleadora ni otorga órdenes directas a éstos, es decir difícilmente podrá desbordar facultades de dirección y disciplina que ni siquiera le han sido conferidas. Además, nunca podrán ser perpetrados por un tercero ajeno a la relación laboral, salvo en el caso del derecho a la vida en el cual la empresa principal sí tiene obligaciones directas, en lo relativo a los accidentes de trabajo y enfermedades profesionales por el deber de protección.

También se señala que «[no] está jurídicamente autorizado para ejercer facultades del empleador la empresa principal respecto de los trabajadores de la empresa contratista o subcontratista que le presta servicios», dificultad que no estaría presente, en cambio, en el caso de las empresas usuarias (Díaz García, 2010: 374-375). ${ }^{10}$

No obstante, es menester aclarar que el artículo 485 del Código del Trabajo establece que dicha afectación a los derechos del trabajador debe ocasionarse respecto

9. De acuerdo con William Thayer y Patricio Novoa, esta facultad de mando comprende la potestad de dirección y el poder disciplinario, es por esta razón que hemos decidido agruparlas de esa manera para permitir una concepción más amplia sobre las facultades del empleador que al ejercerlas darían lugar a una afectación directa a los derechos fundamentales de sus trabajadores (Díaz García, 2010: 371).

10. En este mismo sentido puede entenderse a Caamaño Rojo (2007:189-19o) y Palavecino Cáceres (2014: 35) al decir este último autor sobre las empresas usuarias que: «si se hiciera una interpretación literal, "podría resultar tremendamente peligrosa, ya que haría ilusoria la tutela jurisdiccional de los derechos fundamentales de los trabajadores transitorios frente a la conculcación de estos por la EU [empresa usuaria], lo que transformaría a la disposición del artículo 183-Y del Código del Trabajo en una norma meramente declarativa"», por ejemplo. 
de las cuestiones suscitadas en la relación laboral, con la finalidad de distinguir una eventual vulneración a una garantía constitucional no vinculada propiamente al contexto laboral, como sucedería si ambos sujetos no actuaran en sus calidades de empleador y trabajador. En el caso de un régimen de subcontratación, no olvidemos que la eventual violación a los derechos fundamentales se produce, precisamente, dentro de una modalidad de trabajo en la que interviene la empresa principal para quien se presta los servicios personales, en última instancia.

Retomando la tesis negacionista, se alega que la imposibilidad de ejercer derechos de información y retención, o en términos simples la facultad de fiscalización sobre la empresa contratista o subcontratista, se traduciría en una responsabilidad objetiva en perjuicio de la mandante que opera de forma excepcional y por mandato expreso del legislador. Esta postura ha sido compartida por Urzúa (2018: 287-288), pues afirma:

En la práctica, si se declara la responsabilidad subsidiaria/solidaria de la empresa principal respecto de otras sanciones reguladas en el Código del Trabajo, tal como lo es el recargo legal derivado de la declaración de un despido injustificado de un trabajador de la contratista y/o subcontratista, despido que por lo demás no efectúa, ni incide de forma directa la empresa principal, ni tampoco controla o supervigila esta última, por ello las posturas en esta materia son difíciles de defender en términos absolutos.

El problema en torno a esta arista se reduce a lo que suele afirmarse: «a lo imposible nadie, está obligado», lo que más adelante será refutado con una tesis contrapuesta.

Otro planteamiento dice relación con una aparente desconexión legal entre las normas que determinan la tutela laboral y las del régimen de subcontratación, dictadas estas últimas varios meses después que las primeras. Este criterio pone énfasis en que este vacío en el Código del Trabajo no responde a una cuestión accidental. En otras palabras:

Da la impresión que las normas de subcontratación no existían en aquella época, lo cual es efectivamente cierto, no existían, toda vez que la Ley 20.123 que estableció las normas sobre trabajo en régimen de subcontratación fue publicada nueve meses después de la Reforma Procesal Laboral, circunstancia que suele ser olvidada, pero que ciertamente ayuda a explicar la total desconexión existente entre ambos cuerpos normativos (Sanhueza Torres, 2015: 187).

De igual manera, este criterio será rechazado en la siguiente sección ya que da lugar a un argumento autónomo de la tesis contraria, aunque se haya desarrollado como contrarrespuesta a este razonamiento. Sin embargo, es preciso adelantar que este argumento es controversial, pues omite parcialmente algunos antecedentes históricos que son relevantes en la dictación tanto de la Ley 20.123 como de la Ley 20.087.

Para concluir con los principales criterios de esta postura, decidimos destacar uno 
que consideramos útil en el debate recurrente sobre el rol que juegan los jueces frente a la concepción de los derechos como principios, usualmente entendidos desde la teoría conflictivista de Robert Alexy, y de lo que hoy se denomina neoconstitucionalismo. En tal virtud, para quienes apoyan esta posición, la idea misma de que el tribunal se aparte de una interpretación literal de las normas vinculadas al régimen de subcontratación, permitiendo que la empresa principal pueda responder de forma solidaria y subsidiaria respecto de la indemnización específica de la tutela laboral, resta protagonismo al legislador para dar paso a un juez carente de fundamento democrático. Como lo manifiesta, de alguna manera, Palavecino Cáceres (2014: 44):

Pero si grande ha sido el entusiasmo, grande podría ser también la desilusión. Nuestra propia experiencia respecto de la acción constitucional de protección y la experiencia comparada en materia de tutela judicial de los derechos fundamentales aconsejan un saludable pesimismo. En efecto, la tutela judicial directa de los derechos fundamentales, en lugar de jugar a favor de su protección, termina muchas veces atentando contra las libertades. Nacidos para proteger al individuo frente a la insaciable voluntad de poder de Leviatán, los derechos fundamentales terminan siendo la mejor excusa para que el Estado-Juez restrinja arbitrariamente las libertades de los privados. Por otra parte, la fórmula genere una inquietante disminución del protagonismo de la ley (en definitiva de los órganos deliberativos democráticos) en la tarea delimitadora de los derechos fundamentales y reguladora de los conflictos individuales y su sustitución por el juez (que adolece de un notorio déficit democrático).

Por el contenido de este argumento, es importante añadir algunos puntos en contra que ponen de manifiesto lo cuestionable de entenderlo en términos tales que pudiera bastar por sí mismo para obstaculizar una eventual responsabilidad de la mandante, si se pretendiera. Como se ha dicho, en lo que coinciden ambas tesis es en que existe una laguna respecto de las reglas que generan el presente debate, por lo que, existiendo tal omisión hecha por la función legislativa, es dable traducirla en una habilitación legal hacia el juez para elegir discrecionalmente aquella lectura de las normas que atienda a una interpretación conforme a una norma de rango superior, como sería la carta fundamental, considerando también el principio in dubio pro operario o la regla de la norma más favorable, que resultaría plenamente aplicable en este escenario. Sobre estos dos principios de orden laboral conviene señalar que el principio in dubio pro operario será procedente si se considera que existe un vacío en las normas que regulan la tutela laboral o la subcontratación, de forma independiente, pero si se estima que en su conjunto contienen dicha laguna, entonces operará la regla de la norma más favorable. No hay que olvidar que la primera directriz «se refiere a que si una norma puede entenderse de varias maneras debe preferirse aquella interpretación más favorable al trabajador», mientras que la segunda hace alusión a 
que «en caso de que haya más de una norma aplicable debe optarse por aquella que sea más favorable, aunque no sea la que hubiera correspondido según los criterios clásicos sobre jerarquía de las normas» (Gamonal Contreras, 1998: 132-141). En palabras de Luigi Ferrajoli, la interpretación «siempre es el fruto de una elección práctica respecto de hipótesis interpretativas alternativas» (Díaz García, 2010: 369).

La tutela laboral, así como las reglas que determinan la subcontratación en el código de esta rama, no busca otra cosa que amparar los derechos del trabajador - cualquiera sea la modalidad de trabajo en la que se desempeñe-, estableciendo limitaciones inherentes a la libertad empresarial y a las potestades del empleador, de lo contrario se llegaría al absurdo de que la prestación de servicios bajo subcontratación, al tratarse solo de un contrato civil o comercial entre las empresas, no tendría que estar regulado por el Código del Trabajo, inclusive.

El juzgador, entonces, actuaría dentro de los propios márgenes impuestos por el legislador y en ejercicio de la competencia constitucional que le ha sido atribuida, como es la resolución de conflictos, aplicando las normas conforme a las interpretaciones permitidas y más armónicas con el marco normativo. En este mismo contexto, es conveniente señalar el principio de inexcusabilidad aplicable a los tribunales, que garantiza la tutela judicial efectiva, contenido en el artículo 76 inciso segundo de la Constitución. También se podría aplicar una interpretación sistemática del cuerpo legal, de manera que ambas regulaciones se encuentren debidamente concatenadas en la protección de la dignidad del trabajador.

\section{Tesis que afirma la responsabilidad de la empresa principal}

En esta sección, nos ocuparemos de varios planteamientos que sustentan la tesis de la responsabilidad de la empresa principal desde una óptica constitucional, destacando, además, otros elementos que favorecen a esta postura. Varios de estos argumentos ya fueron expuestos como contrapartida de la tesis negacionista, por lo que aquí nos enfocaremos en aquellos que son sustancialmente independientes. ${ }^{11}$

Para esto, debemos partir de que la constitucionalización del derecho implica que los principios contenidos en la carta fundamental irradian a cada uno de los ámbitos jurídicos, lógicamente respetando las normas e instituciones propias de ellos. Caamaño Rojo (2007: 172) manifiesta:

11. Existen varias sentencias emitidas por diferentes tribunales en los que se hace una interpretación amplia del artículo 183-B del Código del Trabajo, como en la sentencia del caso Urra Carrasco con Productos del Mar Servicios y Construcción Margarita Villegas Ltda. y Ministerio de Obras Públicas, Juzgado de Letras del Trabajo de Puerto Montt, RIT T-11-2016, 11 de mayo de 2016; y la sentencia del caso Guevara con Rendic Hermanos S.A., Juzgado de Letras del Trabajo de Iquique, rol 235-19, 15 de septiembre de 2020 , entre otras. 
De esta manera, el paulatino y decidido proceso de constitucionalización del derecho del trabajo ha contribuido a introducir elementos de juicio que permiten resolver la tradicional tensión entre el poder privado entregado al empleador y el debido respeto de los derechos fundamentales extralaborales del trabajador.

Como consecuencia de la constitucionalización se ha desarrollado el concepto de ciudadanía en la empresa ${ }^{12}$ y se ha estatuido la acción constitucional de rango legal que es la tutela laboral, puesto que ambas dotan a la especialidad de la rama laboral de herramientas prácticas que brindan eficacia a las garantías constitucionales.

Por otro lado, la regulación específica de la subcontratación en el Código del Trabajo tuvo como fundamento la modernización de las diferentes formas contractuales, al tiempo que se reconoció del modo más amplio posible los derechos fundamentales del trabajador. Dicho de otra manera, conjugó la evolución de los modos de producción en el contexto de la globalización, pero sin perder de vista a los trabajadores, sea que estén sujetos a un régimen común de trabajo o que, además de sus empleadores directos, existan otros sujetos beneficiarios de dicha prestación de servicios en la cadena productiva.

Desde esta perspectiva, si la responsabilidad solidaria y subsidiaria de la empresa principal opera respecto de las indemnizaciones laborales y previsionales comunes, ya sea que se recurra a la presunción de un patrimonio más ostentoso con el cual garantizar dicha deuda en favor del trabajador o a la luz del principio de ajenidad, ${ }^{13}$ impedir que ello sea procedente en el caso de la indemnización especial de la tutela laboral no solo va en contra de aquel aforismo que prohíbe distinguir cuando no lo ha hecho el legislador, sino que tampoco respetaría las instituciones de la ciudadanía en la empresa y la tutela laboral, lo que haría eventualmente ineficaces dichas garantías en el contexto de una subcontratación.

La conclusión anterior, dentro del esquema argumentativo que proponemos, está

12. Esta idea es compartida por Ugarte Cataldo (2018: 16). Por otra parte, Gamonal Contreras (2004: 64) afirma respecto de la ciudadanía en la empresa que entre los elementos que le dieron origen se encuentran un factor económico vinculado a la globalización y las políticas neoliberales de occidente (flexibilidad laboral e individualización de las relaciones laborales) y uno relacionado con el avance tecnológico basado en lo que se conoce como sociedad del conocimiento, que ha significado un haz de posibilidades para el empleador, al momento de ejercer su potestad de dirección y eventualmente violar los derechos del trabajador.

13. Como se desprende, por ejemplo, de la sentencia del caso Lara con Concha y Toro, Segundo Juzgado de Letras de Santiago, RIT T-380-2010, 4 de marzo de 2011: «Que además resulta lógico que la principal responda de este tipo de indemnizaciones por cuanto se ha aprovechado de los servicios de los trabajadores de la contratista sin ser considerada empleadora, por lo que su responsabilidad (solidaria o subsidiaria) viene a ser la moneda de cambio de esta concesión legal que lo libera del peso que significa administrativamente tener todos los trabajadores a su cargo, estando además en una inmejorable situación de subrogarse de los derechos del trabajador para repetir contra la contratista». 
atada al objeto de la tutela laboral — que es la protección de derechos fundamentales en favor del trabajador-, de manera que extender sus efectos hacia la empresa principal no implica desconocer que el obligado principal será el contratista o subcontratista, sino asumir su posible incumplimiento, tal como la responsabilidad solidaria y subsidiaria por concepto de indemnizaciones laborales y previsionales, por ejemplo. En este contexto, de no ser factible la reparación pecuniaria que correspondiera, esta garantía jurisdiccional en favor de derechos laborales inespecíficos tampoco operaría como medida de prevención negativa dentro del régimen de subcontratación, como sí lo hace en un esquema ordinario de trabajo. Si bien la reparación de un derecho fundamental no se agota en su resarcimiento económico o compensación, ha sido una medida tutelar expresamente dispuesta por el legislador, que puede ser asumida de forma directa por la mandante en ese supuesto de inobservancia, a diferencia de otras que pudieran ser procedentes, como garantías de no repetición y medidas de satisfacción, entre otras; en otros términos, aunque se diera cumplimiento al resto de las medidas, la reparación integral y, con ello, la garantía misma del derecho conculcado serían solamente parciales.

Negar la posibilidad de responsabilidad de la mandante conllevaría, incluso, a su total indiferencia respecto a sus contratistas o subcontratistas que adopten medidas o acciones que vulneren los derechos de sus trabajadores, pese a que los mismos forman parte de su cadena productiva. Conviene subrayar que este enfoque negacionista impide que las empresas tomen en serio su papel de respeto y promoción activa de los derechos humanos en sus relaciones comerciales, como lo recogen los «Principios rectores sobre las empresas y los derechos humanos: puesta en práctica del marco de las Naciones Unidas para "proteger, respetar y remediar"». Dicho instrumento internacional incorpora un conjunto de directrices que guían el actuar de las diferentes empresas vinculadas en una relación comercial para prevenir o mitigar las consecuencias negativas de acciones $\mathrm{u}$ omisiones de sus actividades o de sus relaciones comerciales sobre los derechos humanos, fundamentalmente a través de los parámetros que determina la debida diligencia. ${ }^{14}$

Por otra parte, buscar la operatividad de dicha indemnización vinculada con la tutela laboral va de la mano con el derecho a una reparación integral, prescrita en el artículo 63 inciso primero de la Convención Americana de Derechos Humanos y aplicable en virtud del artículo 5 inciso segundo de la Constitución, toda vez que si bien el primer responsable del pago es el empleador directo, ante su eventual incumplimiento se permitiría el resarcimiento económico por parte de otro sujeto empresarial que forma parte de igual manera en el régimen de subcontratación.

Es preciso resaltar que, desde la perspectiva constitucional, entendemos esta in-

14. Instrumento aprobado en 2011 por el Consejo de Derechos Humanos de las Naciones Unidas, mediante la Resolución 17/4. 
demnización como una medida de reparación a causa de la vulneración de un derecho fundamental, pues estimamos que el artículo 489 del Código del Trabajo la incorpora al sujetarla a la determinación del juez, según las circunstancias del caso y dentro del margen establecido por el legislador - de seis a once remuneraciones-, sin perjuicio de otras formas de reparación que podría disponer el tribunal como parte de las medidas conservadoras de las cuales dispone. Es importante tener presente que:

El procedimiento de tutela laboral es una modalidad procesal que tiene por objeto conocer de lesiones a ciertos derechos fundamentales de los trabajadores y, en caso [de] que la lesión se conforme, obtener el cese de la conducta antijurídica y medidas reparatorias (Palavecino Cáceres, 2014: 33).

Lo anterior guarda armonía con el mensaje de la Ley 20.087 que, en su parte pertinente, manifestaba:

La sentencia deberá indicar concretamente las medidas tendientes a obtener la reparación de las consecuencias derivadas de la vulneración constatada, incluyendo la posibilidad de que se imponga el pago de una indemnización. ${ }^{15}$

Además, todo esto mantiene una estrecha relación con una interpretación finalista, ${ }^{16}$ propia del ámbito constitucional en lugar de una legal, principalmente considerando los bienes jurídicos que ampara la tutela laboral y el problema que origina el debate existente al respecto.

En este sentido, Riccardo Guastini señala:

La doctrina mayoritaria sostiene que en esta sede no es admisible una interpretación literal ya que muchas de sus disposiciones son formuladas en un lenguaje extremadamente vago, en realidad son principios. Por otro lado, los principios no pueden ser entendidos literalmente pues de hacerlo su efecto es el de privarlos de todo contenido prescriptivo y hacerlo, así, inutilizable en la aplicación del derecho como lo manifiesta (Gamonal Contreras, 2007: 39).

Consideramos, también, que este mismo enfoque ha sido de alguna manera aplicado en lo que se refiere al derecho a la vida y a la integridad de los trabajadores cuando se trata de accidentes laborales y enfermedades profesionales, en los cuales se

15. Mensaje de la Ley 20.087 de 2006.

16. Como afirma Caamaño Rojo (2007: 190) en el caso de las empresas usuarias: «Por lo anterior, creemos que debe efectuarse una interpretación finalista de las disposiciones de los artículos 183-Y y 485 del Código del Trabajo, en el sentido de aceptar plenamente que los trabajadores transitorios puedan hacer valer el amparo particular de este procedimiento especial de derechos fundamentales frente a la $\mathrm{EU}$, en base a las características propias de la relación laboral atípica a que da lugar el suministro de trabajadores». 
ha aceptado la responsabilidad directa de la empresa principal. Caamaño Rojo (2007: 167) señala:

Si cabe destacar de manera somera una verdadera innovación normativa respecto de la subcontratación de bienes y servicios, entonces se debe mencionar el fortalecimiento de la protección de los trabajadores de las empresas contratistas y subcontratistas en materia de seguridad laboral, en orden a establecer una responsabilidad directa de la empresa principal en el resguardo de la vida y salud de estos trabajadores. ${ }^{17}$

Hay autores que indican que la responsabilidad directa de la empresa principal opera por acciones propias y no de terceros - frente a omisiones o acciones que pudieron evitar dicha afectación al trabajador-, de lo que podemos destacar varios aspectos.

Primero, el juzgador en esos casos evalúa las medidas que pudieron ser adoptadas por la empresa principal en concreto, es decir, aquellas que no están señaladas expresamente en el cuerpo legal, sino que son derivadas del deber de seguridad con relación a la vida y la salud de los trabajadores recogida en el artículo 183-E del Código del Trabajo, como sucedería de igual manera con el deber de respeto y promoción de los derechos fundamentales del trabajador. Así también, con base en los principios de igual jerarquía y de interdependencia de los derechos humanos, no tendría cabida la responsabilidad exclusiva de la mandante de ciertas garantías constitucionales, dejando desprotegidos jurídicamente a otros igualmente previstos en la tutela laboral y las normas de la carta fundamental.

Desde un enfoque histórico de las leyes 20.087 y 20.123, estimamos bastante cuestionable la supuesta desconexión que existiría entre las normas relativas a la tutela laboral y las de subcontratación. En el mensaje de la Ley 20.087 se manifestó sobre la vigencia de los derechos fundamentales del trabajador:

Requiere, como condición necesaria, no solo de un reconocimiento material, sino que también y ante todo de mecanismos de tutela jurisdiccional eficaces e idóneos. Como nos señala el insigne jurista italiano Norberto Bobbio, el "problema de fondo relativo a los derechos humanos no es tanto el de justificarlos como el de protegerlos".18

De ahí que se deban evaluar y garantizar el cumplimiento eficaz de todas las medidas de reparación de los derechos que pudieran verse vulnerados, de lo contrario resultaría una acción protectora meramente declarativa, del derecho fundamental que se intenta amparar.

17. Con este argumento, también están de acuerdo Ruay Sáez, Miranda Alarcón, Allende y Correa (2019: 422).

18. Mensaje de la Ley 20.087 de 2006. 
Sin perder de vista lo anterior, durante la tramitación de la Ley 19.759 - que modifica el Código del Trabajo en lo relativo a las nuevas modalidades de contratación, al derecho de sindicación, a los derechos fundamentales del trabajador y a otras materias que indica y fue publicada en octubre de 2001-, la Comisión de Trabajo y Previsión Social de la Cámara de Alta sugirió que el tema de la subcontratación recibiera un tratamiento separado de dicho proyecto, en razón de que se estimaba esencialmente «necesario escuchar a los distintos sectores involucrados en la normativa propuesta, lo que no es posible con la detención y profundidad necesaria ${ }^{19}$, dada la urgencia con la que se despachó el proyecto de la ley. Cabe agregar que en el mensaje con el que ingresó este proyecto se resaltó como uno de sus objetivos el mejoramiento del respeto de los derechos fundamentales de los trabajadores. Por consiguiente, el presidente Ricardo Lagos ingresó un nuevo proyecto, la Ley 20.123:

Acogiendo la petición unánime que en su oportunidad formulara la Comisión de Trabajo y Previsión Social de esa Cámara Alta durante el proceso de aprobación de la Ley 19.759 (reforma laboral) en orden a desagregar esta iniciativa sobre trabajo temporal [...]. Por ello, gran parte de las normas contenidas en él, ya han sido discutidas en diversas instancias del proceso legislativo. Pero se incorporaron en esta oportunidad aquellas adiciones que contribuyeron a una más adecuada y completa regulación de esta importante materia.

De esta manera, la Ley 20.087 fue publicada el 3 de enero de 2006, efectivamente nueve meses antes que la Ley 20.123 como resalta la tesis negacionista, empero están relacionadas en términos materiales en lo que concierne a la protección de derechos del trabajador, pues esta última provenía de una iniciativa legislativa que tenía como propósito el amparo de los derechos del trabajador. En suma, no tiene cabida esa supuesta desconexión legal atendiendo con detalle a la historia de ambas leyes.

A modo de cierre, la justificación detrás de esta tesis, que afirma la responsabilidad de la empresa principal se reduce a la efectividad en el cumplimiento de la indemnización por tutela laboral con ocasión del despido, es la misma que tiene como base proteger los derechos fundamentales del trabajador, sea que se sitúe en una relación de subcontratación o en una ordinaria. Por tanto, los argumentos planteados se dirigen a abrir nuevos enfoques de fundamentación frente a una situación que de hecho ocurre, ya que de no ser el caso, no existirían siquiera fallos que analizar, aunque sobraran ideas por plasmar. Como se manifestó, en última instancia esto guarda armonía con el rol que debe desempeñar toda empresa en cuanto al respeto a los derechos humanos asociados a su actividad comercial, más aún si son beneficiarias últimas de dicha cadena productiva.

19. Segundo Informe de la Comisión de Trabajo y Previsión Social de la Ley 19.759 de 2001. 


\section{Conclusiones}

La falta de una norma expresa que determine la responsabilidad de la empresa principal en el régimen de subcontratación respecto de la indemnización contemplada en el artículo 489 inciso tercero del Código del Trabajo genera una enriquecedora discusión si logramos integrar nuevas perspectivas de análisis, como nos propone el derecho constitucional.

No obstante, es menester delimitar el escenario argumentativo desarrollado por la postura que niega que la mandante pueda responder en el caso de la tutela laboral con ocasión del despido. Un primer razonamiento sostiene que la responsabilidad no es procedente en virtud del texto de las normas que regulan la subcontratación, pues solo dicen relación con las obligaciones laborales y previsionales de dar, que por definición excluyen las de no hacer, como sería el respeto de las garantías consagradas en la Constitución.

Además, se ha hecho una distinción entre las indemnizaciones que surgen en virtud del término del contrato de trabajo, que operan como una suerte de compensación para el trabajador, y la indemnización de la tutela laboral, que constituye una sanción en contra del sujeto que ha vulnerado los derechos fundamentales de la parte más débil de la relación jurídica. Asimismo, esta postura negacionista ha destacado el carácter personalísimo de la afectación de estos derechos, lo que impediría atribuir responsabilidad por los actos que hayan sido ejercidos por terceros, por más que exista entre ellos una relación civil o comercial.

Por otra parte, han añadido que la violación de derechos fundamentales debe producirse como consecuencia del ejercicio de la facultad de mando, inherente de forma exclusiva al empleador. Otro punto que se ha señalado es que daría lugar a una responsabilidad objetiva, pues los derechos de información y retención contemplados en el Código del Trabajo no permitirían controlar esta clase de conductas ejecutadas por parte de los contratistas o subcontratistas. Para concluir, se indicó como un siguiente argumento la aparente desconexión entre la Ley 20.123 y la Ley 20.087.

En contrapartida, independiente de los argumentos que se desarrollaron antes para refutar cada uno de los planteamientos anteriores, se sostuvo que no hay que olvidarse del fenómeno de la constitucionalización del derecho, cuya manifestación en esta rama jurídica ha tenido lugar en la noción de ciudadanía en la empresa y de la acción de tutela laboral. A su vez, esta base conceptual se sintetiza en la operatividad que adquiriría la indemnización que establece dicha acción como una medida de reparación en favor del trabajador en régimen de subcontratación, en el supuesto de que la contratista o subcontratista no pueda cumplir con ello.

El punto precedente se relaciona también con la interpretación conforme, la sistemática y la finalista, propias del ámbito constitucional, dando prevalencia a los bienes jurídicos que resguarda la tutela laboral, junto con las reglas que integran el principio 
protectorio. Lo que no significa de ninguna manera restar importancia al rol del legislador para dárselo al juez, sino más bien comprender el papel que juega este último en un Estado de derecho, partiendo del vacío normativo que se produce entre ambas regulaciones y que debe ser resuelto para que pueda dotarse de eficacia a las normas legales pertinentes. Es decir, se ha otorgado una potestad discrecional por parte del legislador al órgano jurisdiccional para optar por aquella lectura de las reglas laborales más atinente con el ordenamiento jurídico, obligándolo a una adecuada motivación al momento de elegir una y no otra interpretación en el caso en concreto.

Adicionalmente, consideramos que la tesis que afirma la responsabilidad de la empresa principal permite aplicar los principios de igual jerarquía e interdependencia de los derechos humanos, tomando en cuenta que sí existe protección respecto de los derechos a la vida e integridad del trabajador y que optar por la tesis negacionista no lo extendería al resto de derechos amparados bajo la acción de tutela laboral en el régimen de subcontratación. Esta tesis también coadyuva a una postura activa de promoción de los derechos humanos de las empresas en sus relaciones comerciales, además de promover su obligación de respeto, deberes que finalmente corresponden a todo miembro de una comunidad.

Para finalizar, se realizó una breve revisión de los antecedentes materiales que vinculan las leyes que incorporaron la tutela laboral y el régimen de subcontratación en el Código del Trabajo y, con ello, se desplazó el argumento sobre la supuesta desconexión basada en un elemento puramente temporal y accesorio para estos efectos.

Es así como podemos esbozar algunas inquietudes a futuro. Una de ellas se vincula con la naturaleza jurídica sui generis que adquiere la tutela laboral, tomando en cuenta el objeto de su protección y el marco legal en el cual se integra, lo que justamente ocasiona esta clase de discusiones. Otro desafío es la falta de armonía que puede existir entre la ciudadanía en la empresa y la acción de tutela, por cuanto, si bien ambas tienen por finalidad proteger los derechos fundamentales del trabajador constituyéndose en límites para las potestades del empleador, la segunda opera de forma restringida respecto a los derechos detallados en el artículo 489 del Código del Trabajo, por lo cual la primera no ha logrado reflejarse por completo en esta acción. El fundamento de ello radica en su mayoría en argumentos similares - o posiblemente los mismos- que los planteados por la tesis negacionista, que fue desarrollada en el presente trabajo.

\section{Referencias}

CaAmaño Rojo, Eduardo (2007). «La ley de subcontratación y la tutela de los derechos fundamentales de los trabajadores de servicios transitorios». Ius et Praxis, 13 (2): 157-194. Disponible en https://bit.ly/3kHgEVg.

DíAz García, Iván (2010). «Aplicación del procedimiento de tutela de derechos fun- 
damentales en el suministro de trabajadores». Ius et Praxis, 16 (2): 353-382. Disponible en https://bit.ly/3HvpfnE.

Gamonal Contreras, Sergio (1998). Introducción al derecho del trabajo. Santiago: Editorial Jurídica ConoSur.

-. (2004). Ciudadanía en la empresa o los derechos fundamentales inespecíficos. Santiago: Fundación de Cultura Universitaria.

-. (2007). El procedimiento de tutela de derechos laborales. Santiago: LexisNexis.

Manresa, María Fernanda (2016). La acción de tutela de derechos fundamentales en contra de la empresa principal o mandante. Memoria para optar al título de magíster en derecho con mención en derecho privado de la Universidad Austral de Chile.

Martínez Carazo, Piedad (2006). «El método de estudio de caso: Estrategia metodológica de la investigación científica». Pensamiento \& Gestión, (20): 165-193. Disponible en https://bit.ly/3ck5rFy.

Palavecino Cáceres, Claudio (2014). «El procedimiento de tutela de derechos fundamentales del trabajador en Chile». Revista Chilena de Derecho del Trabajo y de la Seguridad Social, 5 (9): 33-45. Disponible en https://bit.ly/3yaFJho.

Puntriano Rosas, César (2011). «Los efectos de la subcontratación en las relaciones laborales: Análisis de la responsabilidad solidaria como mecanismo de tutela». Derecho \& Sociedad, (37): 151-165. Disponible en https://bit.ly/3DpIMUb.

Ruay Sáez, Francisco, Romina Miranda Alarcón, Daniela Allende y Magaly Correa (2019). «Subcontratación y derecho procesal laboral en Chile. Algunos temas de relevancia». En Fábio Barroso y Vanessa De Melo (organizadores), Memórias do III Encontro Latino-americano de Jovens Juristas, Subseção Latino-Americana de Jovens Juristas da Sociedad Internacional de Derecho del Trabajo y de la Seguridad Social. Recife: FASA. Disponible en https://bit.ly/3HvkwCL.

SANHuEZa Torres, Rodrigo (2015). «Un límite a la responsabilidad de la empresa principal en el procedimiento de tutela laboral: Comentario a sentencia de la Ilustrísima Corte de Apelaciones de Concepción». Revista Chilena de Derecho del Trabajo y de la Seguridad Social, 6 (12): 186-195. Disponible en https://bit.ly/3dDzKYy. Ugarte Cataldo, José Luis (2018). Derechos fundamentales, tutela y trabajo. Santiago: Legal Publishing.

UrzúA, Romina (2018). «Tutela de derechos fundamentales y subcontratación». En Fernández, Sebastián; Montenegro, Francisca; Ruay, Francisco; Salinas Jaime y Urzúa, Romina, Subcontratación. Límites de la responsabilidad de la empresa principal. Santiago: Libromar. 


\section{Agradecimientos}

Agradezco a la Agencia Chilena de Cooperación Internacional para el Desarrollo (AGCID) por haberme conferido una beca para desarrollar mis estudios de posgrado en Chile, siendo uno de sus resultados el presente trabajo.

\section{Sobre la autora}

Tatiana Estefania Saltos Hidalgo es abogada de la Pontificia Universidad Católica del Ecuador, becaria Agencia Chilena de Cooperación Internacional para el Desarrollo 2020, en la Pontificia Universidad Católica de Chile. Su correo electrónico es tesaltos@uc.cl. 
La Revista Chilena de Derecho del Trabajo y de la Seguridad Social es una publicación semestral del Departamento de Derecho del Trabajo y de la Seguridad Social de la Facultad de Derecho de la Universidad de Chile, y que tiene por objetivo el análisis dogmático y científico de las instituciones jurídico-laborales y de seguridad social tanto nacionales como del derecho comparado y sus principales efectos en las sociedades en las que rigen.

\author{
DIRECTOR \\ Luis Lizama Portal \\ EDITOR \\ Claudio Palavecino Cáceres \\ SECRETARIO DE REDACCIÓN \\ Eduardo Yañez Monje \\ SITIO WEB \\ revistatrabajo.uchile.cl \\ CORREO ELECTRÓNICO \\ pyanez@derecho.uchile.cl \\ LICENCIA DE ESTE ARTÍCULO \\ Creative Commons Atribución Compartir Igual 4.o Internacional
}

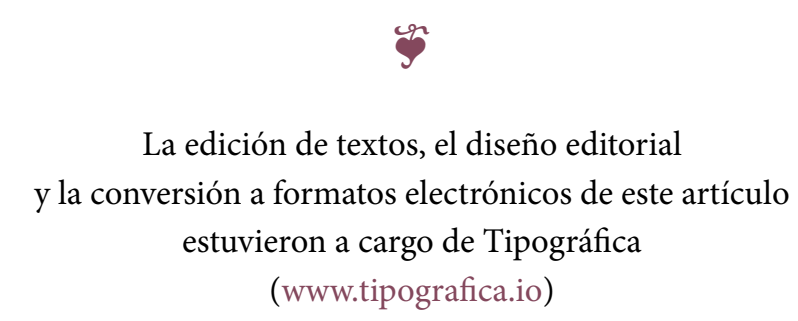

